\title{
Etude préliminaire en vue de la valorisation de vases issues du dragage
}

\author{
Jean-Pierre Troalen*, Delphine Alain et Caroline Sandner** \\ * Maître de Conférences, INPL-ENSG, Laboratoire Environnement, \\ Géomécanique \& Ouvrages, **Elèves-ingénieurs ENSG.
}

Résumé

Si l'identification physico-chimique préalable est reconnue nécessaire vis-àvis des risques de pollution que présentent certains matériaux issus du dragage, le principal problème qu'ils posent reste leur forte teneur en eau pondérale initiale et la quasi-absence de propriétés mécaniques. En raison des faibles quantités utilisées, l'essai de pétrissage MicroHarvard apparaît comme un outil performant quant à la recherche des caractéristiques optimales de densification. Des vases de Seine ont ainsi été densifiées par mélanges avec du ciment et leur comportement mécanique a été étudié en compression uniaxiale pour différents mélanges et différentes teneurs en eau. Les principaux résultats montrent que l'amélioration des propriétés mécaniques n'est pas proportionnelle à la quantité de ciment ajoutée.

\section{Abstract}

When preliminary physico-chemical studies are thought to be necessary for the identification of the risks of pollution that may affect the materials stemming from dredging, the main problem with these materials is their high initial water content and the near absence of mechanical properties. Because of the small amounts used, the MicroHarvard kneading test seems to be a high-performance tool as for the search of optimal characteristics of densification. Some muds from the Seine have been mixed with cement and their mechanical behaviour has been studied in uniaxial compression for different mixtures and different water content. The main results show that the improvement of mechanical properties is not proportionate to the amount of cement added.

\section{Introduction}

Les quantités considérables de matériaux dragués produites annuellement en France nécessitent d'envisager à très court terme leur valorisation et leur réutilisation. Le transport et le stockage provisoire de ces matériaux avant même toute décision de traitement et de valorisation ne peuvent être envisagés sans l'acquisition de propriétés mécaniques minimales, généralement absentes à l'état initial (avant comme après dragage) en raison de leurs très fortes teneurs en eau 
pondérales. Quelle que soit la solution choisie pour aboutir à une densification de ces matériaux (Troalen, 1997), il est nécessaire dans chaque cas d'évaluer la teneur en eau pondérale optimale du matériau intact et par ce biais appréhender le pourcentage d'eau qu'il nous faut abaisser.

Dans les processus classiques de densification et d'amélioration des propriétés mécaniques, les procédés de compactage, tels les essais Proctor, apparaissent comme les plus faciles à mettre en œuvre sauf dans le cas des matériaux dragués en raison même des quantités d'eau interstitielle contenues. C'est pourquoi l'essai de pétrissage représenté par l'essai MicroHarvard (Elsbury et coll., 1988 ; Troalen, 1998) apparaît comme particulièrement intéressant. En effet, il permet d'une part d'obtenir des résultats comparables à ceux des essais Proctor et, d'autre part il ne met en jeu que des quantités réduites de matériaux et peut s'appliquer à tous les types de matériaux dragués. De plus sa réalisation permet la fabrication simultanée d'éprouvettes qu'il est possible de soumettre aussitôt à des essais de compressioni uniaxiale ou triaxiale. Les différents essais peuvent ainsi être multipliés.

\section{Identification minéralogique et physique}

Les vases de Seine qui ont été utilisées pour cette étude ont déjà fait l'objet d'une carâctérisation approfondie (Troalen, Duron et Hagnéré, 1996, 1997 ; Troalen, 1998). Nous n'en donnerons ici que les principaux caractères (Tableau 1).

Deux remarques s'imposent. La première concerne le pourcentage important en argiles qui jouent un rôle de fixateur conséquent vis-à-vis de polluants éventuels. Il conviendra, dans le cas d'un projet de réutilisation des vases, d'identifier la nature de ces polluants et d'en estimer l'importance (Marot, 1997).

$$
\begin{aligned}
& \text { quartz: } 33 \% \text { calcite : } 25 \% \text { dolomite : } 2 \% \text { Feldspaths : } 3 \% \\
& \text { montmorillonite : } 20 \% \quad \text { kaolinite }: 9 \% \text { illite : } 7 \%
\end{aligned}
$$

Tableau 1 : Composition minéralogique des vases de Seine. table 1 : Seine's muds : mineralogy.

La seconde remarque est liée au pourcentage également important en calcite. Son importance vis-à-vis de la fraction silts est relativement exceptionnelle, même si le quartz reste le minéral dominant. Le pourcentage en silts (quartz) apparaît relativement peu élevé pour des vases s.l. et c'est une donnée dont il faudra tenir compte dans le comportement mécanique (Troalen, 1998).

La teneur en eau pondérale des vases étudiées était, à l'état intact (après prélèvement), comprise entre 135 et $163 \%$. L'analyse dimensionnelle montre que la totalité des vases passe au tamis de $100 \mu \mathrm{m}$ ( $40 \%$ entre $90 \mu \mathrm{m}$ et $50 \mu \mathrm{m} ; 5 \%$ entre $50 \mu \mathrm{m}$ et $2 \mu \mathrm{m} ; 55 \%<2 \mu \mathrm{m})$. Les limites d'Atterberg montrent que la limite de liquidité (wl) est située entre 62,3 et 65,1\%, la limite de plasticité (wp) entre 41,9 et $46,4 \%$ et que l'indice de plasticité $\left(\mathrm{I}_{\mathrm{p}}\right)$ est compris entre 18,7 et $20,3 \%$. Les valeurs au bleu de méthylène (VBS) sont comprises entre 3,82 et 3,87 . Ces valeurs 
confirment l'appartenance des vases de Seine à la Classe A des sols fins, sousclasse A2 (LCPC, 1992), c'est-à-dire à des "sables fins argileux, limons argileux et marnes peu plastiques". En effet, pour être en A2, nous devons avoir $2,5<\mathrm{VBS}<$ 6 et un indice de plasticité $12 \%<\mathrm{I}_{\mathrm{p}}<25 \%$.

La mesure de la cohésion intacte non drainée $\left(\mathrm{C}_{\mathrm{U}}\right)$ (scissomètre) montre la quasi absence de propriétés mécaniques des vases de Seine à l'état initial $(0,2 \mathrm{kPa}$ $<\mathrm{C}_{\mathrm{u}}<0,5 \mathrm{kPa}$ ) et cet état physique (densité voisine de $1,3 \mathrm{kN} / \mathrm{m}^{3}$ ) justifie l'application de méthodes visant à leur densification et à l'amélioration de leurs propriétés physiques et mécaniques.

\section{Densification des vases de Seine}

L'objectif est de déterminer la teneur en eau optimale et la densité sèche maximale des vases intactes. Cette opération est réalisée à partir de l'essai de pétrissage MicroHarvard (Elsbury et coll., 1988). Cet essai n'est pas normalisé AFNOR et nous avons utilisé ici la procédure interne MS2-07 mise au point dans notre Laboratoire Environnement, Géomécanique \& Ouvrages (03/1999).

Cet essai consiste à humidifier à des temeurs en eau différentes un matériau préalablement séché, puis de le pétrir selon la procédure d'essai définie. Nous obtenons une courbe Harvard (analogue à la courbe Proctor) qui représente la variation de la masse volumique sèche (en ordonnée) en fonction de la teneur en eau (en abcisse) Sur cette courbe, nous déterminons graphiquement, comme le définit la norme Proctor, la teneur en eau "optimale" et une "densité sèche maximale".

Pour indication, la préparation d'une éprouvette $(\mathrm{h}=7,16 \mathrm{~cm}$; diamètre $=$ $3,30 \mathrm{~cm}$ ) au MicroHarvard demande en moyenne $150 \mathrm{~g}$ d'échantillon de matériau sec (séchage à $105^{\circ} \mathrm{C}$ ). Nous sommes donc loin des quantités nécessaires à la réalisation d'un essai Proctor.

\subsection{Propriétés optimales des vases de Seine intactes}

Un essai de pétrissage MicroHarvard a été réalisé sur des vase de Seine prélevées dans l'avant-port du Havre et préalablement séchées. Cet essai nous a permis de déterminer la teneur en eau optimale et la densité sèche maximale (masse volumique sèche) analogues aux paramètres mesurés habituellement lors de l'essai Proctor. Ces résultats sont mentionnés sur le tableau 2.

\begin{tabular}{|lllll|}
\hline & essai 1 & essai 2 & essai 3 & essai 4 \\
Teneur en eau $(\%)$ & 22,6 & 18,9 & 26 & 32,6 \\
Densité sèche $\left(\mathrm{g} / \mathrm{cm}^{3}\right)$ & 1,46 & 1,29 & 1,46 & 1,29 \\
\hline
\end{tabular}

Tableau 2 : Essai MicroHarvard.

Table 2 : MicroHarvard test.

La teneur en eau optimale déterminée graphiquement (Figure 1) est d'environ $24,5 \%$ pour une densité sèche (masse volumique sèche) de $1,47 \mathrm{~g} / \mathrm{cm}^{3}$. Cette teneur en eau est donc très éloignée de la teneur en eau naturelle (intacte) de $163 \%$. 


\subsection{Comportement des vases après traitement}

Les caractéristiques mécaniques des vases telles qu'elles nous sont apparues après une première tentative de densification par pétrissage sont encore trop faibles pour envisager une utilisation quelconque. En raison des études antérieures entreprises sur ce même matériau (Boutouil, 1998 ; Troalen et al., 1996, 1997 ; Troalen, 1998), nous avons décidé d'améliorer les propriétés mécaniques des vases par l'ajout d'un ciment CPJ 42.5.

Une densification des mélanges vase-ciment et la réalisation d'éprouvettes d'essai est d'abord réalisée par pétrissage au MicroHarvard, à teneurs en eau et en ciment différentes. Les éprouvettes sont conservées sous plastique et stockées en salle climatisée pendant $24 \mathrm{~h}$ avant d'être soumises à des essais de compression uniaxiale. Cette phase est assure aux mélanges vase-ciment un temps suffisant pour les réactions de prise. Elle évite ou réduit considérablement la variabilité observée dans les valeurs des teneurs en eau initiales (après réalisation) des éprouvettes.

\subsubsection{Essais de résistance en compression uniaxiale}

L'évolution de la valeur de la résistance en compression uniaxiale en fonction de la teneur en ciment a été étudiée, d'une part dans des domaines de teneur en eau inférieur et supérieur à celui de la teneur en eau optimale des vases intactes, d'autre part en fonction de la teneur en eau initiale. La vitesse de chargement, à l'aide d'une presse asservie et avec enregistrement continu des paramètres (force, déplacement), était constante et égale à $1 \mathrm{~mm} / \mathrm{mn}$.

La première série d'essais a été conduite avec les teneurs en ciment de $0 \%$, $5 \%, 7,5 \%$ et $10 \%$, pour une teneur en eau initiale du mélange égale à $20 \%$. Les résultats correspondent aux graphes de la figure 1.

La seconde série d'essais reprend les mêmes teneurs en ciment, mais pour une teneur en eau initiale du mélange de $26 \%$. Les résultats sont reportés sur la figure 2 .

La troisième série d'essais met en évidence l'évolution de la résistance en compression uniaxiale des vases intactes en fonction de la teneur en eau (de 18\% à $32 \%$, figure 3 ) et des mélanges vase-ciment précédents, pour $7,5 \%$ de teneur en ciment (figure 4).

Si nous regardons les courbes de résistance en compression données sur la figure 1 , nous remarquons que la valeur de la résistance à la rupture la plus forte correspond à la teneur en ciment la plus élevée. Par contre, en terme de propriétés mécaniques, l'amélioration ne paraît pas proportionnelle à la quantité de ciment ajoutée, en effet à $10 \%$ de ciment résistance à la rupture n'est guère supérieure à celle obtenue pour une teneur en ciment de $7,5 \%$. Par ailleurs, la croissance de la valeur de la contrainte à la rupture est associée à une augmentation du pourcentage de déformation ; cette remarque est complémentaire de l'observation des courbes de compression (avec et sans ciment) qui montrent bien que l'ajout de ciment diminue la plasticité du matériau testé.

La seconde série d'essais de compression uniaxiale (figure 2) a été effectuée à une teneur en eau de mélange supérieure à celle de l'optimum. Nous remarquons que les valeurs maximales de la résistance à la rupture sont inférieures à celles mesurées précédemment (figure 1), mais que la valeur la plus élevée est toujours 


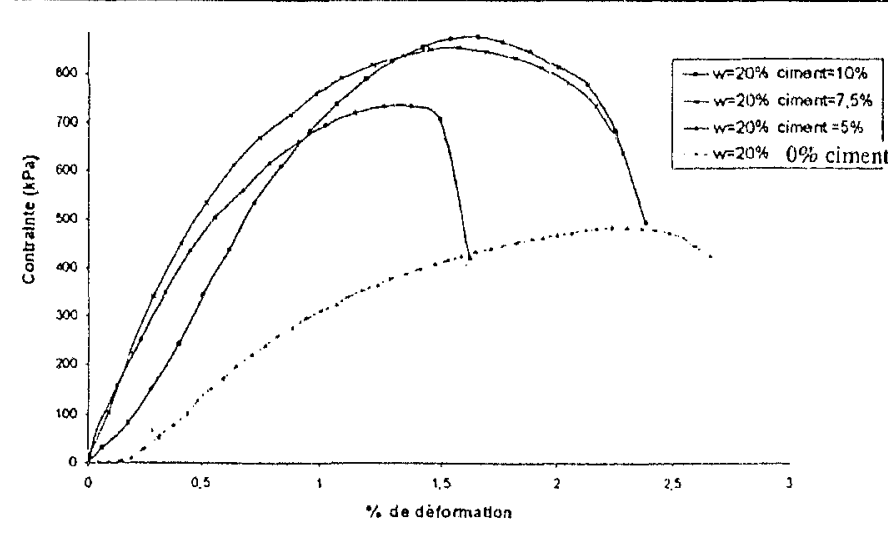

Figure 1 : Essais de résistance en compression uniaxiale $(w=20 \%)$ Figure 1: Uniaxial compression tests $(w=20 \%)$

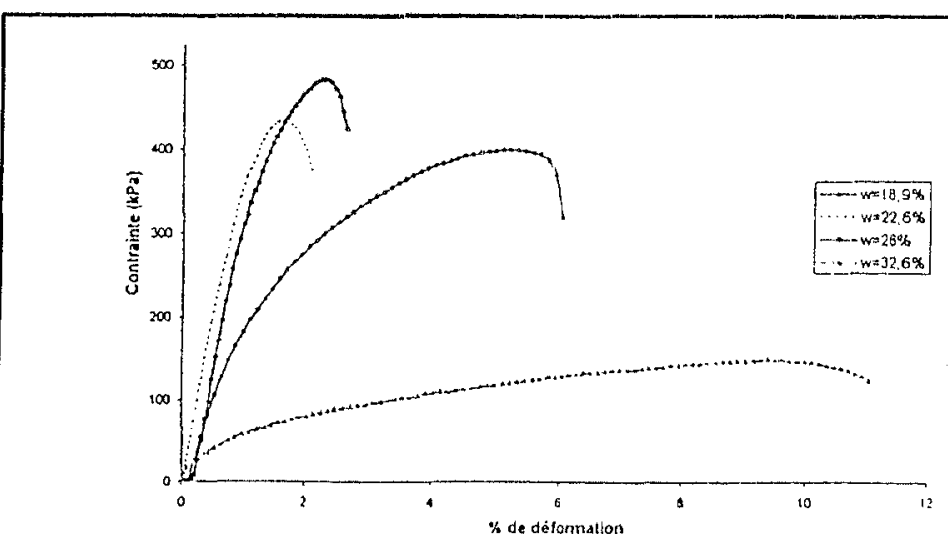

Figure 3 : Essais de résistance en compression uniaxiale (variation $w \%$ ) Figure 3: Uniaxial compression tests (w\% variation)

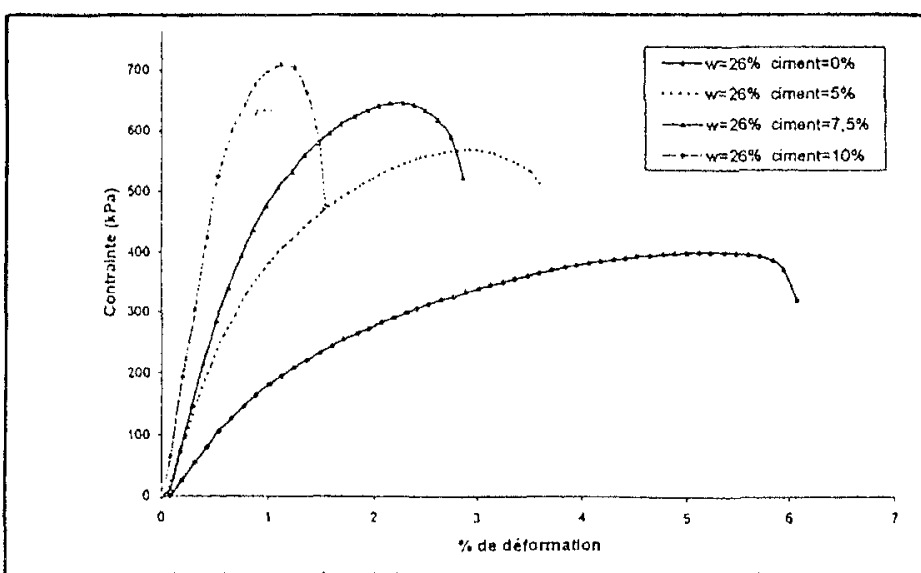

Figure 2 : Essais de résistance en compression uniaxiale $(w=26 \%)$ Figure 2: Uniaxial compression tests $(w=26 \%)$

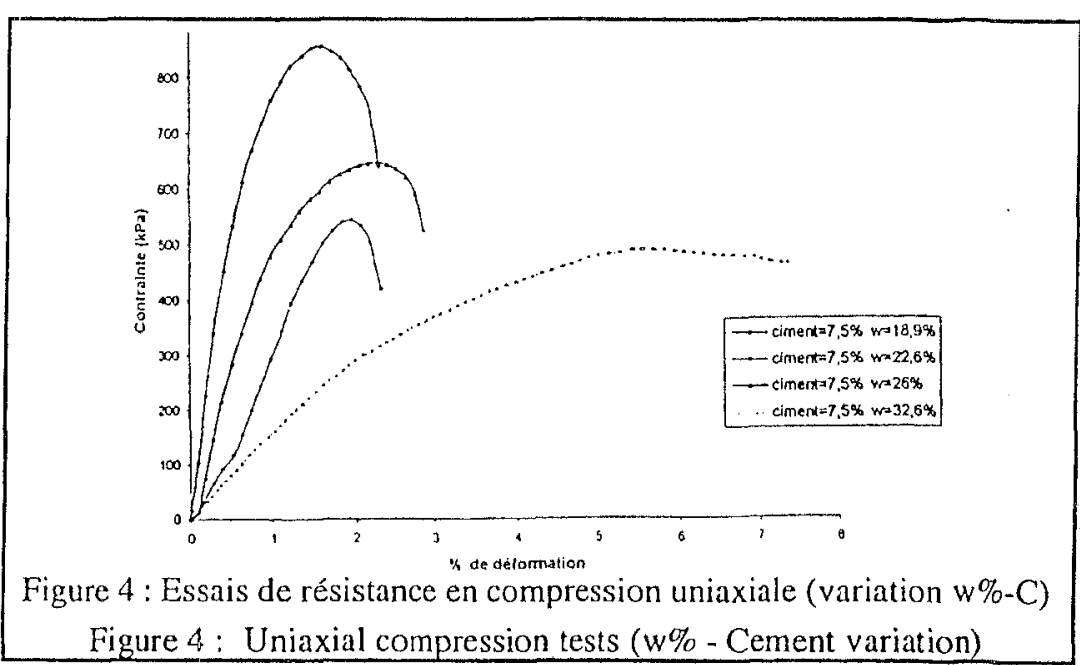


obtenue pour le pourcentage en ciment le plus fort (10\%). De plus, l'accroissement de la teneur en ciment déplace la rupture vers le domaine fragile (le module de Young augmente). La teneur optimale en ciment est ici plus élevée (égale ou supérieure à $10 \%$ ).

La figure 3 nous montre les courbes de résistance en compression des vases pures à différentes teneurs en eau. Ces résultats correspondent aux éprouvettes réalisées lors des essais MicroHarvard précédents (cf § 3.1. et tableau 1). Nous constatons sur les courbes de la figure 3 que le comportement mécanique de la vase est bien conditionné par la teneur en eau initiale. Pour les teneurs en eau inférieures à la teneur en eau optimale $(24,5 \%)$ la rupture est obtenue en domaine fragile.

Pour les teneurs en eau supérieures à la teneur en eau optimale, le comportement des vases tend vers un domaine plastique et la valeur de la résistance à la rupture devient de moins en moins élevée. Les propriétés mécaniques les meilleures sont donc bien celles obtenues pour les densité sèche maximale et teneur en eau optimale de la vase définies lors de l'essai de pétrissage MicroHarvard.

La figure 4 (mélanges vase- $7,5 \%$ ciment, teneurs en eau croissantes) montre les mêmes comportements que précédemment. La différence essentielle réside dans les valeurs plus élevées de la résistance à la rupture en raison de l'ajout de ciment.

Ces différents essais démontrent l'importance de la connaissance de la teneur en eau initiale des mélanges vis-à-vis du choix du pourcentage d'ajout en ciment, et la nécessité de définir au préalable les caractéristiques optimales des vases pures. En effet, quelque soit la teneur de l'ajout de ciment, la contrainte maximale à la rupture $\mathrm{e}$

\subsubsection{Mesures de la cohésion au scissomètre}

L'évolution de la cohésion intacte non drainée et de la cohésion résiduelle des vases et mélanges vase-ciment a été étudiée à l'aide d'un scissomètre pour différentes teneurs en eau initiales et différents pourcentages de ciment (tableau 3).

\begin{tabular}{|c|c|c|c|c|c|c|}
\hline teneur en eau $(\%)$ & $20 \quad 22$ & 25 & 35 & 40 & 50 & 60 \\
\hline cohésion intacte $(\mathrm{kPa})$ & $0,38 \quad 2,07$ & 4,62 & 3,81 & 3,47 & 3,35 & 0,09 \\
\hline cohésion résiduelle $(\mathrm{kPa})$ & $0,190,77$ & 1,33 & 1,30 & 1,28 & 1,20 & 0,09 \\
\hline teneur en eau $=20 \%$ & ciment $(\%)$ & & 0 & 5 & 7,5 & 10 \\
\hline \multicolumn{3}{|c|}{ cohésion intacte non drainée $(\mathrm{kPa})$} & 0,38 & 0,89 & 1,98 & \\
\hline \multicolumn{3}{|c|}{ cohésion résiduelle $(\mathrm{kPa})$} & 0,19 & 0,25 & 0,70 & \\
\hline teneur en eau $=22 \%$ & \multicolumn{2}{|l|}{ ciment $(\%)$} & 0 & 5 & 7,5 & 10 \\
\hline \multicolumn{3}{|c|}{ cohésion intacte non drainée $(\mathrm{kPa})$} & 2,07 & 2,39 & 2,50 & 3,72 \\
\hline \multicolumn{3}{|c|}{ cohésion résiduelle $(\mathrm{kPa})$} & 0,77 & 0,79 & 0,85 & 1,06 \\
\hline teneur en eau $=25 \%$ & ciment $(\%)$ & & 0 & 5 & 7,5 & 10 \\
\hline \multicolumn{3}{|c|}{ cohésion intacte non drainée $(\mathrm{kPa})$} & 4,62 & 5,47 & 6,33 & 7,31 \\
\hline \multicolumn{3}{|c|}{ cohésion résiduelle $(\mathrm{kPa})$} & 1,33 & 1,63 & 2,78 & 3,35 \\
\hline
\end{tabular}

Tableau 3 : Evolution de la cohésion en fonction de la teneur en eau et du pourcentage de ciment.

Table 3 : Evolution of cohesion for different water and cement purcents. 
Nous constatons que les valeurs des cohésions intacte non drainée et résiduelle de la vase non traitée sont les plus élevées pour une teneur en eau de $25 \%$ qui correspond pratiquement à la teneur en eau optimale $(24,5 \%)$ mesurée au MicroHarvard. Les valeurs de la cohésion augmentent rapidement jusqu'à ce seuil, puis diminuent progressivement.

En ce qui concerne les mesures effectuées sur les mélanges vase-ciment, les valeurs des cohésions intactes non drainée et résiduelle augmentent en fonction du pourcentage d'ajout de ciment et sont également maximum pour les mélanges préparés à la teneur en eau optimale de $25 \%$. L'accroissement de la valeur de la cohésion intacte non drainée est très sensible dès le seuil des $8 \%$ de ciment.

Il est également à remarquer que les valeurs de la cohésion intacte et de la cohésion résiduelle augmentent en fonction du temps et sont généralement stabilisées à leur valeur maximale au bout de 3 h 30 environ. Ce qui doit correspondre à la fin de la réaction de prise vase-ciment.

\section{Conclusion}

Cette étude préliminaire de vases issues du dragage met systématiquement en évidence la nécessité d'appréhender la valeur de sa teneur en eau optimale. En effet, du strict point de vue du comportement, les propriétés mécaniques des vases non traitées ou traitées par du ciment sont optimales pour cette teneur en eau proche des $25 \%$, quel que soit le pourcentage d'ajout en ciment.

De plus, la connaissance préalable de la teneur en eau optimale des vases est une donnée intéressante vis-à-vis d'une opération de séchage partiel, c'est-à-dire en fait, l'estimation de la quantité d'eau interstitielle qu'il est nécessaire d'éliminer avant une réutilisation ou un traitement, et surtout en vue d'une densification.

D'autre part, s'il apparaît nettement que l'ajout de ciment améliore les propriétés physiques et mécaniques des vases issues du dragage, il semble que cette amélioration ne soit pas proportionnelle à la quantité de ciment ajoutée. Un optimum de résistance à là rupture se situerait aux alentours de $7,5 \%$ de ciment.

Cette constatation demande à être confirmée. En effet, dans l'étude que nous avons réalisé, il n'a pas été tenu compte dans l'interprétation des résultats de l'influence exacte de la nature des constituants initiaux, en particulier le rôle éventuel de variations des pourcentages en silts (quartz), en carbonates et en argiles s.s. Nous avons vu lors de l'identification minéralogique que dans le cas présent le pourcentage en calcite était relativement élevé (25\%) par rapport au pourcentage de silts $(33 \%)$ généralement plus conséquent dans ce type de vase. Il apparaît évident que ces pourcentages, liés eux-mêmes au pourcentage et à la nature de la phase argileuse s.s., peuvent, dans le cas d'une variation de leurs teneurs, ne plus avoir la même réaction de prise lors d'un mélange avec le ciment.

Dans tous les cas, la réaction de prise ciment-vase reste fortement dépendante de la quantité d'eau interstitielle disponible et du pourcentage de ciment ajouté.

Nous avons vu dans cette étude préliminaire (densification) qu'en fait, il semblerait que les propriétés optimales soient celles obtenues pour la teneur en eau optimale MicroHarvard, ce qui est confirmé par les mesures de la cohésion intacte non drainée au scissomètre. 


\section{Références}

BOUTOUIL M. (1998) - Traitement des vases de dragage par solidification/stabilisation à base de ciment et additifs, Thèse de Doctorat de l'Université du Havre, France, 197 p.

ELSBURY et coll. (1988) - Field and laboratory testing of compacted soil liner, US EPA, Cincinnati, USA, pp. 240-256.

L.C.P.C. (1992) - Réalisation des remblais et des couches de forme, Guide Technique, fascicules $1 \& 2,75732$ Paris cedex 15, France.

MAROT F. (1997) - Caractérisation et traitement de sédiments de dragage contenant des polluants métalliques, Thèse de Doctorat de l'Université du Havre, France, $330 \mathrm{p}$.

TROALEN JP., DURON C. \& HAGNERE C. (1996) - Traitement de vases de l'estuaire de la Seine, Revue Française de Géotechnique, 77, pp. 31-36.

TROALEN JP., DURON C. \& HAGNERE C. (1997) - Caractérisation et traitement de vases d'estuaire, Actes du $1^{\mathrm{er}}$ Congrés International de l'AIGI "La géologie de l'ingénieur et l'environnement", Athènes (Grèce), 23-27/06/97, pp. 1635-1642, Balkema, Rotterdam. ISBN 90-5410-877-0.

TROALEN JP. (1997) - Amélioration et densification de vases d'esiuaire, Process of the $3^{\text {rd }}$ International Conference on Ground Improvment Geosystem :: Densification and Reinforcement, Londres (UK), 3-6/06/97, Thomas Telford publishing.

TROALEN JP. (1998) - Comportement et réutilisation de vases d'estuaire (Seine) traitées par un limon quaternaire, Process of the $8^{\circ}$ Congress of the International Association of Engineering Geology and Environment, Vancouver (Canada), 2125/09/98, pp. 3875-3882, Balkema, Rotterdam. ISBN 90-5410-990-4.

TROALEN JP. (1998) - Vases issues du dragage : solidification et comportement, Actes du Colloque MAGI'50 "Les matériaux argileux et la géologie de l'ingénieur", ENSG-Nancy (France), 21-22/09/98, pp. 77-84, Sciences de la Terre Editeur ASGA, ISBN 2-85555-053-X. 